\title{
Transient Monoparesis Due to Brainstem and Thalamic Herpes Simplex 2 Encephalitis
}

\author{
David Whitney ${ }^{\mathrm{a}, \mathrm{b}}$, EJ Gettings ${ }^{\mathrm{a}}$, Troy Desai ${ }^{\mathrm{a}}$, Thomas Scott ${ }^{\mathrm{a}}$
}

\begin{abstract}
PCR testing for herpesvirus infections has broadened our understanding of the full spectrum of central nervous system disorders seen as a result of herpesvirus infections. Herpesvirus infections of the brainstem are rare, and are usually due to HSV-1 or varicella zoster. Clinicians need to be aware of an ever increasing variety of presentations for these neurological syndromes. Over time, through case reports, we may see patterns emerge concerning the clinical presentations of particular herpesvirus family members. We add to a group of rare reports of brainstem involvement in HSV-2 infection (more commonly due to HSV-1).
\end{abstract}

Keywords: Magnetic resonance imaging; Herpes simplex virus 1; Herpes simplex virus 2; Brainstem; Thalamus

\section{Introduction}

Herpes simplex virus (HSV) is one of the most common pathogens causing encephalitis in adults, with an incidence of 1 case per 250,000 to 500,000 per year [1]. Though it most commonly affects the temporal lobes, herpes simplex encephalitis can localize to other areas of the brain, and there are several case reports of HSV encephalitis involving the brainstem. In these cases, symptoms usually include neuroophthalmologic findings and cranial nerve deficits [2]. Less commonly, focal motor weakness can develop. As in HSV encephalitis, HSV-1 predominates in brainstem encephalitis due to herpesviruses, but a few cases have been caused by

\footnotetext{
Manuscript accepted for publication June 6, 2013

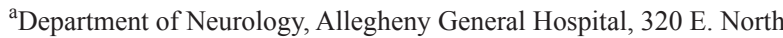
Ave., Pittsburgh PA 15212, USA

${ }^{\mathrm{b} C o r r e s p o n d i n g ~ a u t h o r: ~ D a v i d ~ W h i t n e y, ~} 320$ E. North Ave., Pittsburgh PA 15212, USA. Email: david.m.whitney@temple.edu
}

doi: http://dx.doi.org/10.4021/jmc1319w
HSV-2 [2]. In this case, we describe a patient who developed transient left lower extremity weakness due to HSV-2 encephalitis affecting the brainstem and thalami.

\section{Case Report}

A 62-year-old woman was admitted to the hospital for an 8 -week history of headache and 1-week history of left leg weakness, leading to walking difficulty and multiple falls. Her past medical history was remarkable for chronic migraine headaches since age 16, diabetes mellitus type 2, and Crohn's disease. Headaches were initially accompanied by fever and chills. Outpatient evaluation 5 weeks prior to admission resulted in discharge from the emergency room. A second evaluation 3 weeks prior to admission resulted in a brief hospital stay and treatment with ceftriaxone and ciprofloxacin. She then developed left leg weakness and was admitted to an outside hospital one week before transfer to our care.

Two days prior to transfer to our facility, cerebrospinal fluid (CSF) examination revealed cloudy fluid with an opening pressure of $16 \mathrm{~cm} \mathrm{H}_{2} \mathrm{O}$, elevated glucose $(86 \mathrm{mg} / \mathrm{dL})$, elevated protein $(192 \mathrm{mg} / \mathrm{dL})$, markedly elevated white blood cell count ( 1535 cells $/ \mu \mathrm{L} ; 100 \%$ lymphocytes), and red blood cell count of $1 \mathrm{cell} / \mu \mathrm{L}$. Additional studies performed prior to transfer to our care including $\mathrm{x}$-rays of her lumbar spine, left pelvis, hip, and knee, magnetic resonance imaging (MRI) of the cervical and lumbar spine and peripheral white blood cell count $(5.4 \mathrm{k} / \mu \mathrm{L})$ were all within normal limits.

On admission to our hospital neurologic examination revealed mild to moderate proximal and distal left lower extremity weakness. Sensation in the left lower extremity was slightly diminished to light touch and vibration and reflexes were decreased. Mental status was normal. MRI of the brain revealed small focal areas of restricted diffusion in a patchy distribution involving the bilateral pons, midbrain, and posterior thalami (Fig. 1). These areas also demonstrated signal hyperintensity on fluid-attenuated inversion recovery sequencing and did not enhance with intravenous gadolinium contrast. Repeat CSF studies on hospital day 7 revealed clear fluid with an opening pressure of $16 \mathrm{~cm} \mathrm{H}_{2} \mathrm{O}$, decreased glu- 


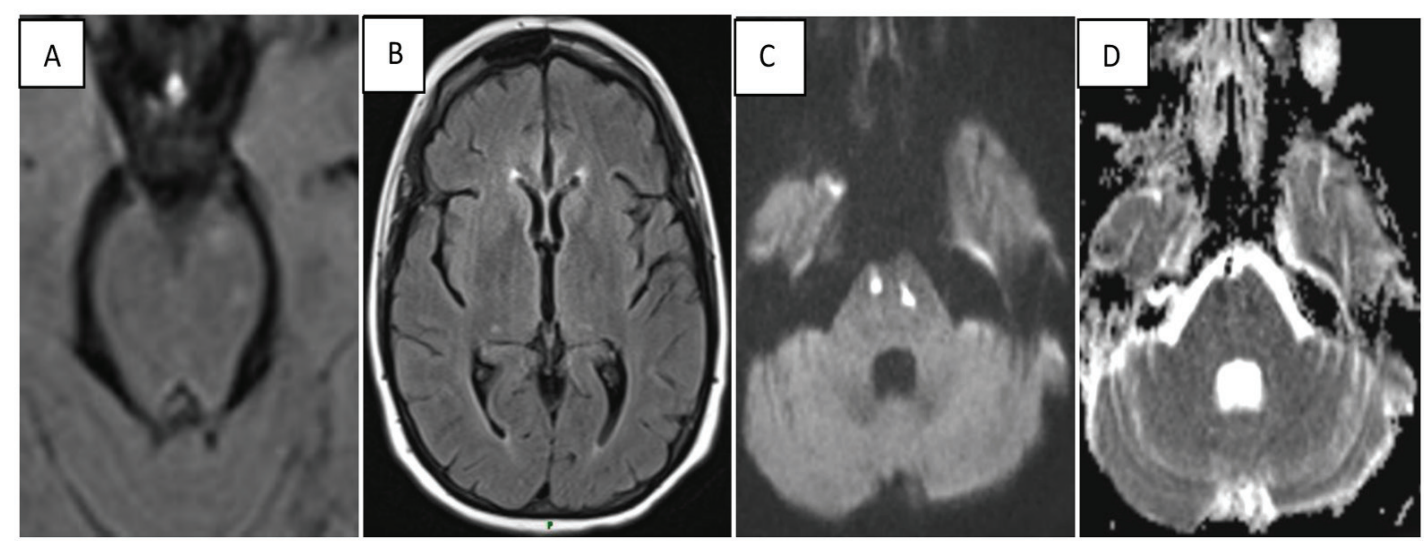

Figure 1. Multifocal lesions of the pons, midbrain, and bilateral thalami, some of which exhibited restricted diffusion. (A), FLAIR image, multifocal midbrain lesions. (B), FLAIR image, bithalamic lesions. (C, D), Diffusion weighted imaging demonstrating areas in pons of restricted diffusion and corresponding ADC abnormality, consistent with acute cytotoxic injury.

cose $(19 \mathrm{mg} / \mathrm{dL})$, elevated protein $(162 \mathrm{mg} / \mathrm{dL})$, improved but still elevated white blood cell count (103 cells/ $\mu \mathrm{L} ; 87 \%$ lymphocytes, $13 \%$ monocytes), and red blood cell count of 173 cells $/ \mu$ L. Polymerase chain reaction (PCR) analysis of this sample was positive for HSV-2 DNA. PCR studies for HSV-1, Epstein Barr virus, cytomegalovirus, varicella zoster, West Nile virus (WNV) and lymphocytic choriomeningitis virus (LCMV) were negative. Additionally, serum HIV antibodies, rapid plasma reagent (RPR), serum angiotensin converting enzyme (ACE), and Lyme antibody were negative, as well as a vasculitis panel including p-ANCA, c-ANCA, anti-myeloperoxidase antibodies and proteinase-3 antibodies. Antinuclear antibody (ANA) titer was weakly positive at 1:20 and anti-Ro/SS-A antibody was borderline positive. CSF cytology, CSF flow cytometry and computed tomography (CT) scans of her chest, abdomen, and pelvis demonstrated no abnormalities.

At the time of discharge the patient was walker dependent. After 2 weeks inpatient rehabilitation followed by 3 months outpatient physical therapy, the patient required a cane except for short distances, exhibiting steady improvement.

\section{Discussion}

We add to a group of rare reports of brainstem involvement in HSV-2 infection (more commonly HSV-1) [2]. Even fewer cases of thalamic involvement with either HSV-1 or HSV-2 have been described, most of which were pediatric [3]. Indeed, this pattern of multifocal thalamic and pontine lesions has been reported only once previously, wherein the HSV subtype was not determined [4].

The clinical and radiographic differential diagnosis in this case included inflammatory disorders such as small vessel vasculitis, CNS lymphoma or lymphomatous meningitis, other infections such as WNV or LCMV, chronic lymphocytic inflammation with pontine perivascular enhancement responsive to steroids (CLIPPERS), and neurosarcoidosis. Differential diagnosis would have included multiple sclerosis more prominently in a younger patient, but was unlikely here. This list of possibilities for patients presenting with these non-specific clinical and radiologic findings thus includes disorders which are difficult to confirm, in some cases requiring long-term follow-up or even brain biopsy. Positive real-time PCR for HSV-2, which has a specificity of $95.4 \%$, was essential for diagnosis in this case [5].

Magnetic resonance angiography likely rules out medium or large vessel vasculitis in our patient, however, without autopsy or biopsy for tissue analysis, it is not possible for us to rule out infarctions related to small vessel vasculitis as the substrate of the clinical and radiographic features in this case. Brainstem locations of encephalitis due to herpes virus infections are particularly difficult to distinguish versus a vascular substrate of injury. Patients presenting with sudden onset of stroke-like syndromes after herpes virus infections are frequently assumed to have small vessel vasculitis, which may occur within a day or several weeks after apparent infection. Since both cytotoxic and vasogenic edema may occur as a result of brainstem encephalitis, diffusionweighted MRI with apparent diffusion coefficient mapping is useful for prognosis, but is not able to distinguish between vasculitic versus encephalitic-induced injury. Additionally, some patients have been shown to have both a vasculitis and encephalitis simultaneously. Fortunately, for clinicians, it is assumed that treatments with antiviral agents are possibly effective in either scenario, and are generally recommended when a diagnosis is established by PCR in cerebrospinal fluid in patients with disabling or progressive symptoms. Steroids could be effective as a treatment for brainstem encephalitis as well in severe cases, although high-class evidence for this theory is lacking. In our opinion, it is reasonable to monitor 
patients presenting with mild symptoms without pharmacologic intervention, but high-level evidence for this strategy is also lacking.

Though rare, HSV-2 infection should be considered in patients with meningoencephalitis and MRI findings indicative of multifocal lesions involving the brainstem and thalami. Clinicians should consider examining CSF PCR for herpesviruses in this setting, allowing for expedient diagnosis and treatment.

\section{Grant Support}

None.

\section{Disclosures}

All authors declare there are no conflicts of interest and no financial disclosures related to this case.

\section{References}

1. Whitley RJ, Gnann JW. Viral encephalitis: familiar infections and emerging pathogens. Lancet. 2002;359(9305):507-513.

2. Livorsi D, Anderson E, Qureshi S, Howard M, Wang YF, Franco-Paredes C. Brainstem encephalitis: an unusual presentation of herpes simplex virus infection. $\mathrm{J}$ Neurol. 2010;257(9):1432-1437.

3. De Tiege X, Rozenberg F, Burlot K, Gaudelus J, Ponsot $\mathrm{G}$, Heron B. Herpes simplex encephalitis: diagnostic problems and late relapse. Dev Med Child Neurol. 2006;48(1):60-63.

4. Duarte J, Argente J, Gutierrez P, Catalan MJ, Cabello A, Claveria LE. Herpes simplex brainstem encephalitis with a relapsing course. J Neurol. 1994;241(6):401-403.

5. Gaeta A, Verzaro S, Cristina LM, Mancini C, Nazzari C. Diagnosis of neurological herpesvirus infections: real time PCR in cerebral spinal fluid analysis. New Microbiol. 2009;32(4):333-340. 